\title{
Robust Low Altitude Behavior Control of a Quadrotor Rotorcraft Through Sliding Modes
}

\author{
Mehmet Önder Efe ${ }^{1}$
}

\begin{abstract}
This paper gives the full dynamical model of a commercially available quadrotor rotorcraft and presents its behavior control at low altitudes through sliding mode control. The control law is very well known for its robustness against disturbances and invariance during the sliding regime. The plant, on the other hand, is a nonlinear one with state variables are tightly coupled. The simulations have shown that the algorithm successfully drives the system towards the desired trajectory with bounded control signals.
\end{abstract}

\section{Unmanned Aerial Vehicles (UAVs)}

A UAV is a flying robot having its own power supply, having no human pilot and maintaining the flight through an appropriate scheduling of aerodynamic forces either autonomously or by remote control. The UAV systems are capable of being invisible to radars and of performing formation flight. With such properties, a UAV system is cheap enough to sacrifice and powerful enough to carry sensors, camera and communication systems and lightweight weapons. More importantly, a UAV can maintain the flight beyond the limits of a human pilot.

Such kind of small scale and highly versatile systems are used in a wide spectrum of applications. For instance, collecting information (imaging, pursuit, searching, video acquisition and reconnaissance), security, surveillance, control (smuggling prevention), targeting, meteorologic and agricultural applications, traffic management and steering, telemetry (remote sizing) and crisis management after natural disasters are some examples at a first glance.

Accomplishing these high level missions with UAV systems is critically dependent upon the performance at low level command and control schemes. This fact has made the design, prototyping, implementation and manufacturing of autopilot systems a growing industry. The choice of the autopilot for a UAV system may depend upon the mission statement yet, regardless of the mission statement, the vehicle must be robust enough to cope with the difficulties of the operating environment. Sliding mode control systems are very well known for their robustness against disturbances and invariance during the sliding mode. The technique is known also as Variable Structure Control (VSC) as the system during the sliding regime operates in a predefined subset of the phase space.

This work is supported by TOBB ETÜ BAP Program (Contract No: ETÜ BAP 2006/04)

${ }^{1}$ Corresponding Author, TOBB Economics and Technology University, Department of Electrical and Electronics Engineering, Sögütözü, Ankara, Turkey, E-mail: onderefe@ieee.org, Phone: +90-312-292-4064, Fax: +90-3122924091
VSC scheme is well-known for its robustness to uncertainties and disturbances. Conceptually, the controller design in this framework is based on the nominal representation of the system about which the bounds of the uncertainties are assumed to be available. The decision mechanism operates on the basis of switching on the different sides of a decision boundary, which is called the sliding hypersurface [1], [2], [3], and the goal of the design is to enforce the error vector toward this hypersurface during the reaching phase. Once the error vector is confined to the sliding hypersurface, it obeys the behavior imposed by the set of equations describing the hypersurface, i.e. sliding mode starts and the error vector converges to origin. The control strategy is therefore called Sliding Mode Control (SMC) in the related literature, [1], [2], [3]. During the sliding mode, the control system becomes insensitive to the disturbances and uncertainties unless the decision mechanism violates the physical limits for maintaining the sliding motion.

SMC strategy has been applied successfully in a wide variety of design problems ranging from the control of motion control systems, and chemical processes to the control of chaotic systems. Hung et al., [1], review the control strategy for linear and nonlinear systems and discusses the design for systems represented in canonical forms. Another systematic examination of SMC approach is presented in [4], in which the practical aspects of SMC design are assessed for both continuous-time and discrete-time cases and a special consideration is given to the finite switching frequency, limited bandwidth actuators and parasitic dynamics. Misawa discusses the SMC design for discrete-time systems in [5] for the linear case and in [6] for the nonlinear case with unmatched uncertainties, Sabanovic et al. [7] elaborate the chattering free SMC design, Bartolini et al. [8] formulate the chattering-free SMC for MIMO systems, and Erbatur et al. [9] investigate the robustness properties of SMC technique on a 2-DOF direct drive SCARA robot.

Extensive range of application domains of the SMC scheme with robustness properties motivate us to design the low level control laws for the quadrotor rotorcraft system considered in this paper and by some other researchers. For example Castillo et al. [10], [11] have performed real time experiments and assessed the performance of a nonlinear controller. In [12], classical PID controller is considered and model based design is experimented. Hanford et al., [13], present a simple closed loop equipped with MEMS sensors and PIC based processing unit. Hoffman et al., [14], achieve the formation control by SMC technique and focuse on collision and obstacle avoidance by extracting the state variables with a Kalman filter. Vision based control of the quadrotor rotorcraft system is 
studied in [15], which exploits the Moiré patterns, and in [16], which utilizes double cameras. Camlica dwells on a linear quadratic controller in [17], and Waslander puts an emphasis on the insufficiency of classical control methods and proposes the integral SMC associated with reinforcement learning to achieve multi agent control. In [19], vehicle stabilization based on the backstepping technique is presented with successful results.

The current SMC design problem is involved with coupled and highly nonlinear dynamics, noisy observations and demanding performance requirements. The organization of the paper is as follows: The dynamic model of the vehicle is presented in the next section, the SMC technique is presented in the third section, behavior control is discussed next, simulation results and concluding remarks are given at the end of the paper.

\section{The Dynamic Model of The Vehicle}

A sketch of the quadrotor rotorcraft system studied in this study is shown in Fig. 1, where the Euler angles and the cartesian coordinate frame are shown. The equations of motion are given in (1) and the values of some variables seen are tabulated in Table I.

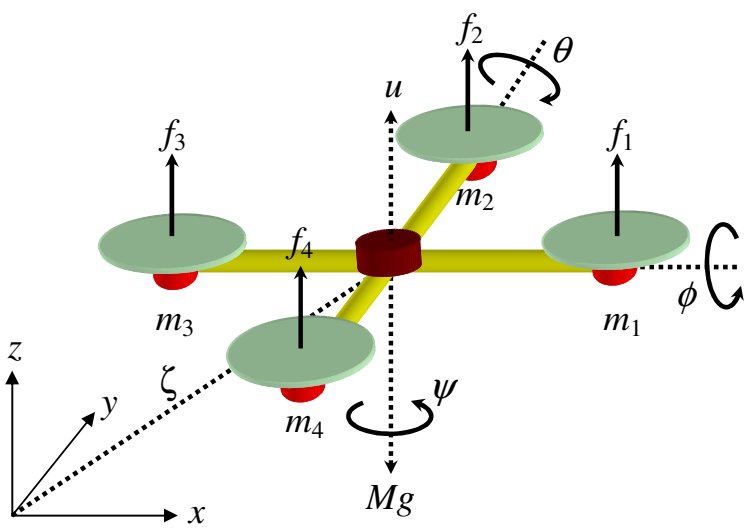

Fig. 1. General view of the quadrotor rotorcraft system

$$
\begin{aligned}
M \ddot{x} & =-u s_{\theta} \\
M \ddot{y} & =u c_{\theta} s_{\phi} \\
M \ddot{z} & =u c_{\theta} c_{\phi}-M g \\
\ddot{\psi} & =\tilde{\tau}_{\psi} \\
\ddot{\theta} & =\tilde{\tau}_{\theta} \\
\ddot{\phi} & =\tilde{\tau}_{\phi}
\end{aligned}
$$

where $s \theta$ is an abbreviation for $\sin \theta$ while $c \theta$ stands for $\cos \theta$.

$$
\tilde{\tau}=\left(\begin{array}{c}
\tilde{\tau}_{\psi} \\
\tilde{\tau}_{\theta} \\
\tilde{\tau}_{\phi}
\end{array}\right)=\mathbb{J}^{-1}(\tau-C(\eta, \dot{\eta}) \dot{\eta})
$$

Here $\eta=\left(\begin{array}{ccc}\psi & \theta & \phi\end{array}\right)^{\mathrm{T}}, \mathbb{J}(\eta)=T_{\eta}^{\mathrm{T}} \mathbf{I} T_{\eta}$ and

$$
\begin{gathered}
T_{\eta}=\left(\begin{array}{ccc}
-s_{\theta} & 0 & 1 \\
c_{\theta} s_{\phi} & c_{\phi} & 0 \\
c_{\theta} c_{\phi} & -s_{\phi} & 0
\end{array}\right) \\
\mathbf{I}=\left(\begin{array}{ccc}
I_{x x} & 0 & 0 \\
0 & I_{y y} & 0 \\
0 & 0 & I_{z z}
\end{array}\right)=\left(\begin{array}{ccc}
I_{x x} & 0 & 0 \\
0 & I_{x x} & 0 \\
0 & 0 & 2 I_{x x}
\end{array}\right)
\end{gathered}
$$

The Coriolis and centripetal vector denoted by $C(\eta, \dot{\eta})$ is defined as below and computed as given by (8).

$$
\begin{gathered}
C(\eta, \dot{\eta})=\dot{\mathbb{J}}-\frac{1}{2} \frac{\partial}{\partial \eta}\left(\dot{\eta}^{\mathrm{T}} \mathbb{J}\right) \\
\mathbb{J}=I_{x x}\left(\begin{array}{ccc}
1+c_{\theta}^{2} c_{\phi}^{2} & -c_{\theta} s_{\phi} c_{\phi} & -s_{\theta} \\
-c_{\theta} s_{\phi} c_{\phi} & 2-c_{\phi}^{2} & 0 \\
-s_{\theta} & 0 & 1
\end{array}\right)
\end{gathered}
$$

$$
\dot{\mathbb{J}}=I_{x x}\left(\begin{array}{ccc}
\dot{\theta} s_{2 \theta} c_{\phi}^{2}+\dot{\phi} s_{2 \phi} c_{\theta}^{2} & \dot{\theta} s_{\theta} s_{\phi} c_{\phi}-\dot{\phi} c_{2 \phi} c_{\theta} & \dot{\theta} c_{\theta} \\
\dot{\theta} s_{\theta} s_{\phi} c_{\phi}-\dot{\phi} c_{2 \phi} c_{\theta} & \dot{\phi} s_{2 \phi} & 0 \\
\dot{\theta} c_{\theta} & 0 & 0
\end{array}\right)
$$

$$
\begin{aligned}
& C_{1,1}=C_{1,2}=C_{1,3}=0 \\
& C_{2,1}=I_{x x}\left(\dot{\psi} c_{\phi}^{2} s_{2 \theta}+\dot{\theta} s_{\phi} c_{\phi} s_{\theta}-\dot{\phi} c_{\theta}\right) \\
& C_{2,2}=I_{x x} \dot{\psi} s_{\phi} c_{\phi} s_{\theta} \\
& C_{2,3}=-I_{x x} \dot{\psi} c_{\theta} \\
& C_{3,1}=-I_{x x}\left(\dot{\psi} c_{\theta}^{2} s_{2 \phi}+\dot{\theta} c_{\theta} c_{2 \phi}\right) \\
& C_{3,2}=-I_{x x}\left(\dot{\psi} c_{\theta} c_{2 \phi}-\dot{\theta} s_{2 \phi}\right) \\
& C_{3,3}=0
\end{aligned}
$$

where $I_{x x}=I_{y y}=m \ell^{2}, I_{z z}=2 m \ell^{2}$. Model inputs and the aerodynamic forces $\left(f_{i}\right)$ created by each propeller are related to each other as described below.

$$
\begin{aligned}
\tau_{\psi} & =\sum_{i=1}^{4} \tau_{m_{i}} \\
\tau_{\theta} & =\left(f_{3}-f_{1}\right) \ell \\
\tau_{\phi} & =\left(f_{2}-f_{4}\right) \ell \\
u & =\sum_{i=1}^{4} f_{i}
\end{aligned}
$$

In above, $f_{i}=k \omega_{i}^{2}$ and $k>0$ is a motor gain, $\omega_{i}$ denotes the angular velocity of $i$ th motor. (See [10], [11] for details).

TABLE I

PHYSICAL PARAMETERS OF THE QUADROTOR ROTORCRAFT

\begin{tabular}{|c|c|c|}
\hline \hline$m_{i}$ & Motor weight & $0.08 \mathrm{~kg}$ \\
\hline$m_{b}$ & Battery weight & $0.20 \mathrm{~kg}$ \\
\hline$M$ & Total weight of the vehicle & $0.52 \mathrm{~kg}$ \\
\hline$\ell$ & Distance from motors to COG & $0.205 \mathrm{~m}$ \\
\hline$g$ & Gravitational acceleration & $9.81 \mathrm{~m} / \mathrm{s}^{2}$ \\
\hline \hline
\end{tabular}




\section{An Overview of Sliding Mode Control}

Assume that the plant under control has the structure described in (10), where $\xi$ and $\dot{\xi}$ are the states, and $\delta$ is the control input.

$$
\ddot{\xi}=\Phi(\xi, \dot{\xi})+\Psi(\xi, \dot{\xi}) \delta
$$

where $\Psi(\xi, \dot{\xi}) \neq 0$. The design problem is to enforce the behavior of the system states towards the desired trajectories, which are known. Denote the reference trajectories by $\xi_{r}$ and $\dot{\xi}_{r}$ and the tracking errors by $e_{\xi}=\xi-\xi_{r}$ and $\dot{e}_{\xi}=\dot{\xi}-\dot{\xi}_{r}$.

The crux of the SMC scheme is the definition of a sliding manifold, along which the sliding motion is to take place. This quantity is denoted by $s$ and is defined as below

$$
\begin{aligned}
s & =\left(\frac{\mathrm{d}}{\mathrm{d} t}+\lambda\right) e_{\xi} \\
& =\dot{e}_{\xi}+\lambda e_{\xi}
\end{aligned}
$$

where $\lambda>0$ is the slope of the sliding line ${ }^{1}$. If a control law enforces the trajectories in the phase space such that $s=0$ holds true, then the errors converge asymptotically to origin as prescribed by $\dot{e}_{\xi}=-\lambda e_{\xi}$, whose solution is $e_{\xi}(t)=e_{\xi}(0) \mathrm{e}^{-\lambda t}$.

In order to demonstrate stability, adopt the Lyaponuv function candidate given as

$$
V=\frac{1}{2} s^{2}
$$

The time derivative of the Lyapunov function in (12) can be computed as follows

$$
\begin{aligned}
\dot{V} & =s \dot{s} \\
& =s\left(\ddot{e}_{\xi}+\lambda \dot{e}_{\xi}\right) \\
& =s\left(\ddot{\xi}-\ddot{\xi}_{r}+\lambda \dot{e}_{\xi}\right) \\
& =s\left(\Phi(\xi, \dot{\xi})+\Psi(\xi, \dot{\xi}) \delta-\ddot{\xi}_{r}+\lambda \dot{e}_{\xi}\right)
\end{aligned}
$$

We would like to have $\dot{V}=s \dot{s} \leq-\sigma|s|$ with $\sigma$ being a positive constant. Dropping the arguments of the functions $\Phi$ and $\Psi$, and equating $\Phi+\Psi \delta-\ddot{\xi}_{r}+\lambda \dot{e}_{\xi}$ to $-\sigma \operatorname{sgn}(s)$ and solving for $\delta$ yields

$$
\delta=\frac{1}{\Psi}\left(-\sigma \operatorname{sgn}(s)-\lambda \dot{e}_{\xi}+\ddot{\xi}_{r}-\Phi\right)
$$

which ensures $\dot{s}=-\sigma \operatorname{sgn}(s)$ and $\dot{V}=-\sigma|s|$ is achieved in the closed loop. The control law in (14) has two properties.

- If an initial condition, say $\left(e_{\xi}(0), \dot{e}_{\xi}(0)\right)$ is not on the sliding manifold characterized by $s=0$, it is forced towards the manifold. In other words, the sliding manifold is an attractor and the regime until it is reached is called the reaching mode.

- If a trajectory is trapped into the sliding manifold, the system in the closed loop behaves exactly as how the

\footnotetext{
${ }^{1}$ In the current problem the system dynamics is a second order one, therefore the sliding manifold is a line. In general, $\lambda$ is mentioned as the slope parameter determining the speed of convergence during the sliding regime.
}

sliding regime prescribes. During this regime, the closed loop control system becomes insensitive to disturbances to the extent allowed by the design, and this mode is known as the sliding mode.

With such a control law, one naturally questions the selection of $\sigma>0$. Assume the plant given in (10) is a nominal plant, on which the sliding control law is based. If the real plant has uncertainties that enter the right hand side of (10) as below;

$$
\ddot{\xi}=\Phi(\xi, \dot{\xi})+\Psi(\xi, \dot{\xi}) \delta+\Delta(\xi, \dot{\xi}, t)
$$

then the application of the control signal computed for the nominal plant would yield the following result

$$
\dot{s}=-\sigma \operatorname{sgn}(s)+\Delta(\cdot)
$$

Clearly if $\sigma>\sup _{\xi, \dot{\xi}, t}|\Delta(\xi, \dot{\xi}, t)|$ then $s \dot{s}<0$ is ensured. The practical interpretation of this is as follows: For large $\sigma$, the result stipulates that larger uncertainties are tolerable however one has to consider the system specific details, e.g. time constants to determine the best $\sigma$ since a large value of $\sigma$ will require very fast hitting the sliding manifold as formulated by $t_{h} \leq \frac{|s(0)|}{\sigma}$, the proof of which is straightforward.

In the practical applications of SMC systems, the original sign function is smoothed by utilizing the approximation $\operatorname{sgn}(s) \approx \frac{s}{|s|+\varepsilon}$, where $\varepsilon>0$. Since the sliding mode entails $s \approx 0$, the noise in the observed quantities becomes highly effective and the controller can generate unnecessarily large control signals. This is known as the chattering in the related literature, [3]. Utilizing the above approximation introduces a boundary layer and eliminates the undesired chattering phenomenon significantly, [3]. This paper adopts the same strategy in computing the sign of the quantity $s$. In the next section, we present the design of SMC for the considered UAV system.

\section{Control of the Vehicle Behavior}

The control of the vehicle behavior is scrutinized under three subtitles as discussed below. The underlying idea is to compute the desired value of the Euler angles and quickly drive these angles to their desired values. In Fig. 2, the general structure of the control system is shown, where the reference angle generation is followed by the angle controllers thereby resulting in desired behavioral response.

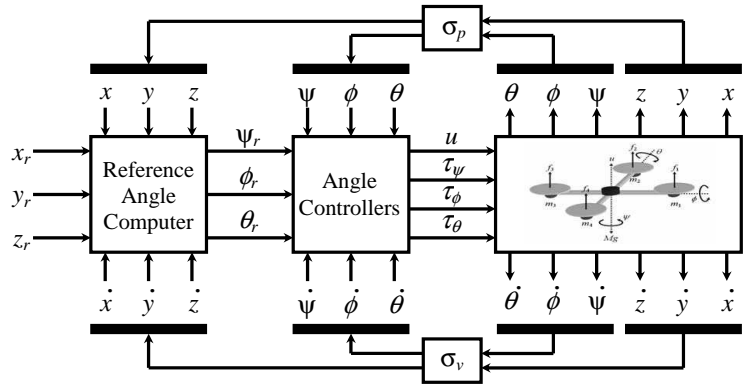

Fig. 2. The block diagram of the control system structure 


\section{A. SMC of the Yaw Angle $(\psi)$ and Altitude $(z)$}

Denote the desired altitude by $z_{r}$, the desired velocity in $z$-direction by $\dot{z}_{r}$ and the desired acceleration by $\ddot{z}_{r}$. Define $e_{z}:=z-z_{r}, \dot{e}_{z}:=\dot{z}-\dot{z}_{r}$ and $s_{z}:=\dot{e}_{z}+\lambda_{z} e_{z}$.

With these definitions, the controller postulated below will drive the vehicle to the desired altitude.

$$
u=M \frac{\ddot{z}_{r}-\lambda_{z} \dot{e}_{z}-\sigma_{z} \frac{s_{z}}{\left|s_{z}\right|+\varepsilon}-\mu_{z} s_{z}+g}{c_{\theta} c_{\phi}}
$$

The control law above is accompanied by the following selection with $\psi_{r}=0$.

$$
\tilde{\tau}_{\psi}=-6 \dot{\psi}-9\left(\psi-\psi_{r}\right)
$$

The SMC law in (17) and the stabilizing law in (18) result in the maintenance of the desired altitude.

\section{B. SMC of the Roll Angle ( $\phi)$ and $y$-Position}

Denote the desired position in $y$-direction by $y_{r}$, the desired velocity by $\dot{y}_{r}$ and the desired acceleration by $\ddot{y}_{r}$. Define $e_{y}:=$ $y-y_{r}, \dot{e}_{y}:=\dot{y}-\dot{y}_{r}$ and $s_{y}:=\dot{e}_{y}+\lambda_{y} e_{y}$. Define the reference roll angle value as

$$
\phi_{r}:=\tan ^{-1}\left(\frac{\ddot{y}_{r}-\lambda_{y} \dot{e}_{y}-\sigma_{y} \frac{s_{y}}{\left|s_{y}\right|+\varepsilon}-\mu_{y} s_{y}}{\ddot{z}_{r}-\lambda_{z} \dot{e}_{z}-\sigma_{z} \frac{s_{z}}{s_{z} \mid+\varepsilon}-\mu_{z} s_{z}+g}\right)
$$

Clearly the control input $u$ in (17) keeps the desired altitude and the behavior in $z$-direction obeys $\dot{s}_{z}=-\sigma_{z} \frac{s_{z}}{\left|s_{z}\right|+\varepsilon}-\mu_{z} s_{z}$. As soon as the transient regime in $z$ direction and $\psi$ angle ends, the dynamic behavior in $y$-direction is governed by

$$
\ddot{y}=\left(\ddot{z}_{r}-\lambda_{z} \dot{e}_{z}-\sigma_{z} \frac{s_{z}}{\left|s_{z}\right|+\varepsilon}-\mu_{z} s_{z}+g\right) \tan \phi
$$

Obviously for $\phi \equiv \phi_{r}$, we would have $\dot{s}_{y}=-\sigma_{y} \frac{s_{y}}{\left|s_{y}\right|+\varepsilon}-\mu_{y} s_{y}$, then one could drive $\phi \rightarrow \phi_{r}$ as quickly as possible and would ensure the stability in $y$-direction too. The control of the roll angle is achieved by choosing

$$
\tilde{\tau}_{\phi}=-6 \dot{\phi}-9\left(\phi-\phi_{r}\right)
$$

\section{SMC of the Pitch Angle ( $\theta)$ and $x$-Position}

Denote the desired position in $x$-direction by $x_{r}$, the desired velocity by $\dot{x}_{r}$ and the desired acceleration by $\ddot{x}_{r}$. Define $e_{x}:=$ $x-x_{r}, \dot{e}_{x}:=\dot{x}-\dot{x}_{r}$ and $s_{x}:=\dot{e}_{x}+\lambda_{x} e_{x}$. Define the reference pitch angle value as

$$
\theta_{r}:=\tan ^{-1}\left(-\frac{\ddot{x}_{r}-\lambda_{x} \dot{e}_{x}-\sigma_{x} \frac{s_{x}}{\left|s_{x}\right|+\varepsilon}-\mu_{x} s_{x}}{\ddot{z}_{r}-\lambda_{z} \dot{e}_{z}-\sigma_{z} \frac{s_{z}}{\left|s_{z}\right|+\varepsilon}-\mu_{z} s_{z}+g} \cos \phi\right)
$$

The choice of $u$ in (17) makes the behavior in $x$-direction as follows:

$$
\ddot{x}=\left(\ddot{z}_{r}-\lambda_{z} \dot{e}_{z}-\sigma_{z} \frac{s_{z}}{\left|s_{z}\right|+\varepsilon}-\mu_{z} s_{z}+g\right) \frac{\tan \theta}{\cos \phi}
$$

If $\theta \equiv \theta_{r}$, we would have $\dot{s}_{x}=-\sigma_{x} \frac{s_{x}}{\left|s_{x}\right|+\varepsilon}-\mu_{x} s_{x}$, then one could drive $\theta \rightarrow \theta_{r}$ as quickly as possible and would ensure the stability in $x$-direction too. The control of the Euler angle $\theta$ is achieved by setting

$$
\tilde{\tau}_{\theta}=-6 \dot{\theta}-9\left(\theta-\theta_{r}\right)
$$

Briefly, in order to achieve a desired response in the cartesian space, as illustrated in Fig. 2, the desired values for the Euler angles are computed and the orientation of the UAV is driven to those particular values which eventually drives the vehicle to the target position in cartesian coordinate system.

\section{Simulation Studies}

Two flight scenarios are considered in the simulations. In the first scenario, the reference trajectories of the vehicle change only in one direction while the other two coordinates are maintained at a constant value. This results in movements along the vertices of rectangular volumes in the cartesian space. The second scenario illustrates the results obtained when the quadrotor rotorcraft system is desired to move along a continuously changing trajectory designed for takeoff and landing in turn.

In Table II, the parameters of the SMC law and the simulations are summarized. One should note that for such applications the selection of the best parameter set is a matter of the design specifications as well as the capabilities of the vehicle under investigation. After a short period of fine tuning by trial and error, we have fixed the values to the tabulated values.

TABLE II

SIMULATION PARAMETERS

\begin{tabular}{|c|c|c|}
\hline \hline$\Delta t$ & Simulation stepsize & 0.25 sec. \\
\hline$T$ & Simulation time (Flight time) & 1300 sec. \\
\hline$\sigma_{p}$ & Variance of positional noise & 0.001 \\
\hline$\sigma_{v}$ & Variance of velocity noise & 0.001 \\
\hline$\sigma_{x}, \sigma_{y}, \sigma_{z}$ & Reaching law parameter & 0.010 \\
\hline$\mu_{x}, \mu_{y}, \mu_{z}$ & Reaching law parameter & 0.500 \\
\hline$\lambda_{x}, \lambda_{y}, \lambda_{z}$ & Slope parameters & 1.000 \\
\hline$\varepsilon$ & Sign function smoothing par. & 0.050 \\
\hline \hline
\end{tabular}

The results with the first scenario are illustrated in Fig. 3, where the UAV system follows the reference trajectory very precisely. The control signals produced to observe this result are not depicted due to the space limit but they have been observed to be smooth enough. In the simulations, we have assumed that the actuators are able to respond quickly and accurately, and we have not enforced limits on the control signals.

In the simulations, all velocities and cartesian positions $(x(0), y(0)$ and $z(0))$ are taken zero initially. The initial values of the Euler angles are $\psi(0)=0.1 \mathrm{rad} ., \theta(0)=0.2 \mathrm{rad}$. and $\phi(0)=-0.1 \mathrm{rad}$. and the obtained results have been found satisfactory.

With the same initial conditions and controller settings, the reference profile is changed to the one given below. 


$$
\begin{gathered}
x_{r}(t)=\left\{\begin{array}{cc}
0 & t<200 \mathrm{sec} . \\
\frac{t-200}{6} \sin \left(\frac{2 \pi t}{400}\right) & 200 \leq t<800 \mathrm{sec} . \\
\frac{1300-t}{5} \sin \left(\frac{2 \pi t}{400}\right) & t \geq 800 \mathrm{sec} .
\end{array}\right. \\
y_{r}(t)=\left\{\begin{array}{cc}
0 & t<200 \mathrm{sec} . \\
\frac{t-200}{6} \cos \left(\frac{2 \pi t}{400}\right) & 200 \leq t<800 \mathrm{sec} . \\
\frac{1300-t}{5} \cos \left(\frac{2 \pi t}{400}\right) & t \geq 800 \mathrm{sec} .
\end{array}\right. \\
z_{r}(t)=\left\{\begin{array}{cc}
100 \tanh \left(\frac{t}{50}\right) & t<200 \mathrm{sec} . \\
100 & 200 \leq t<800 \mathrm{sec} . \\
\frac{1300-t}{5} & t \geq 800 \mathrm{sec} .
\end{array}\right.
\end{gathered}
$$

The results obtained with these command signals are shown in Fig. 4. Clearly the vehicle takes off and reaches an altitude of 100 meters, then starts navigating at this altitude by following a circular trajectory and lands at the origin, where the flight had started. The trajectory tracking ability is found to be very promising in this scenario too. Clearly, the simultaneous changes in the command signals causes a difficulty for the controller presented, yet, it displays certain degrees of robustness against such difficulties and the adverse effects of the observation noise are alleviated successfully.

\section{CONCLUSIONS}

This paper presents the design of sliding mode controllers for low altitude behavior control of a quadrotor rotorcraft system. The assumptions made during the design were the unsaturated controls, negligible weather disturbances (wind gust etc.) and reasonably fast actuation periphery. The paper gives the dynamic model of the vehicle and presents a hierarchical control scheme that drives the vehicle to its desired altitude, then to desired $y$ position and finally the desired $x$ position. The simulations carried out justify the claims, i.e. the controllers perform very well in the first type of flight scenario, where only one of the coordinates is changing. The results obtained with the second type of flight trajectory are found to be very accurate and this result is attributed to the robustness property of sliding mode control systems.

The contribution of this work to the existing body of literature is the presentation of a fairly simple yet robust control strategy for a highly nonlinear unmanned aerial vehicle. The future work of the author contains real time justification of the presented control law.

\section{ACKNOWLEDGEMENTS}

The author gratefully acknowledges the help of Dr. Pedro Castillo and TOBB ETÜ librarians.

This work is supported by TOBB ETÜ BAP Program (Contract No: ETÜ BAP 2006/04).

\section{REFERENCES}

[1] J.Y. Hung, W. Gao and J.C. Hung, "Variable Structure Control: A Survey," IEEE Trans. on Industrial Electronics, 40, pp.2-22, 1993.

[2] Utkin, V.I. Sliding Modes in Control Optimization. Springer Verlag, New York, (1992).

[3] Slotine, J.-J. E. and Li, W. Applied Nonlinear Control, Prentice-Hall, New Jersey, 1991.
[4] K.D. Young, V.I. Utkin and Ü. Özgüner, “A Control Engineer's Guide to Sliding Mode Control," IEEE Trans. on Control Systems Technology, 7, pp.328-342, 1999.

[5] E.A. Misawa, "Discrete-Time Sliding Mode Control: The Linear Case," Trans. of the ASME, Journal of Dynamic Systems, Measurement, and Control, 119, pp.819-821, 1997.

[6] E.A. Misawa, "Discrete-Time Sliding Mode Control for Nonlinear Systems With Unmatched Uncertainties and Uncertain Control Vector," Trans. of the ASME, Journal of Dynamic Systems, Measurement, and Control, 119, pp.503-512, 1997.

[7] A. Sabanovic, N. Sabanovic, K. Jezernik and K. Wada, "Chattering Free Sliding Modes," Proc. of the 3rd Workshop on Variable Structure Systems and Lyapunov Design, pp.143-148, Napoly, Italy, 1994.

[8] G. Bartolini, A. Ferrera, E. Usai, and V.I. Utkin, "On Multi-Input Chattering-Free Second-Order Sliding Mode Control," IEEE Trans. on Automatic Control, 45, pp.1711-1717, 2000.

[9] K. Erbatur, O. Kaynak, and A. Sabanovic, "A Study on Robustness Property of Sliding Mode Controllers: A Novel Design and Experimental Investigations," IEEE Trans. on Industrial Electronics. 46, pp.1012-1018, 1999.

[10] P. Castillo, R. Lozano ve A.E. Dzul, "Stabilization of a Mini Rotorcraft with Four Rotors", IEEE Control Systems Magazine, pp.45-55, December 2005 .

[11] P. Castillo, R. Lozano ve A.E. Dzul, Modelling and Control of Mini Flying Machines, Springer Verlag, 2005.

[12] S. Bouabdallah, A. Noth ve R. Siegwart, "PID vs LQ Control Techniques Applied to an Indoor Micro Quadrotor," Proc. of 2004 IEEE/RSJ Int. Conf. on. Intelligent Robots and Systems, September 28 - October 2, Sendai, Japan, 2004.

[13] S.D. Hanford, L.N. Long ve J.F. Horn, "A Small Semi-Autonomous Rotary-Wing Unmanned Air Vehicle (UAV)," AIAA2005-7077.

[14] G. Hoffmann, D.G. Rajnarayan, S.L. Waslander, D. Dostal, J.S. Jang, C.J. Tomlin, "The Stanford testbed of Autonomous Rotorcraft for Multi Agent Control (STARMAC)," Proc. of The 23rd Digital Avionics Systems Conference (DASC'04), October 24-28, Salt Lake City, Utah, USA, 2004.

[15] G.P. Tournier, M. Valentiy ve J.P. Howz, "Estimation and Control of a Quadrotor Vehicle Using Monocular Vision and Moiré Patterns," AIAA Guidance, Navigation, and Control Conference and Exhibit, August 2124, Keystone, Colorado, USA, AIAA2006-6711, 2006.

[16] E. Altu, J.P. Ostrowski, C.J. Taylor, "Quadrotor Control Using Dual Camera Visual Feedback," Proc. of the 2003 IEEE Int. Conf. on Robotics \& Automation, September 14-19, Taipei, Taiwan, s.4294-4299, 2003.

[17] F.B. amlca, "Demonstration of a Stabilized Hovering Platform for Undergraduate Laboratory," Yksek Lisans Tezi, Orta Dou Teknik niversitesi, Ankara, Trkiye, 2004.

[18] S.L. Waslander, G.M. Hoffmann, J.S. Jang ve C.J. Tomlin, "MultiAgent Quadrotor Testbed Control Design: Integral Sliding Mode vs. Reinforcement Learning," 2005 IEEE/RSJ Int. Conf. on Intelligent Robots and Systems, August 2-6, Edmonton, Alberta, Canada, s.468473, 2005 .

[19] T. Hamel, R. Mahony, R. Lozano ve J. Ostrowski, "Dynamic Modelling and Configuration Stbilization for an X4-Flyer," 15th IFAC Triennial World Congress, Barcelona, Spain, 2002. 
Desired (Solid) Position and Vehicle Position
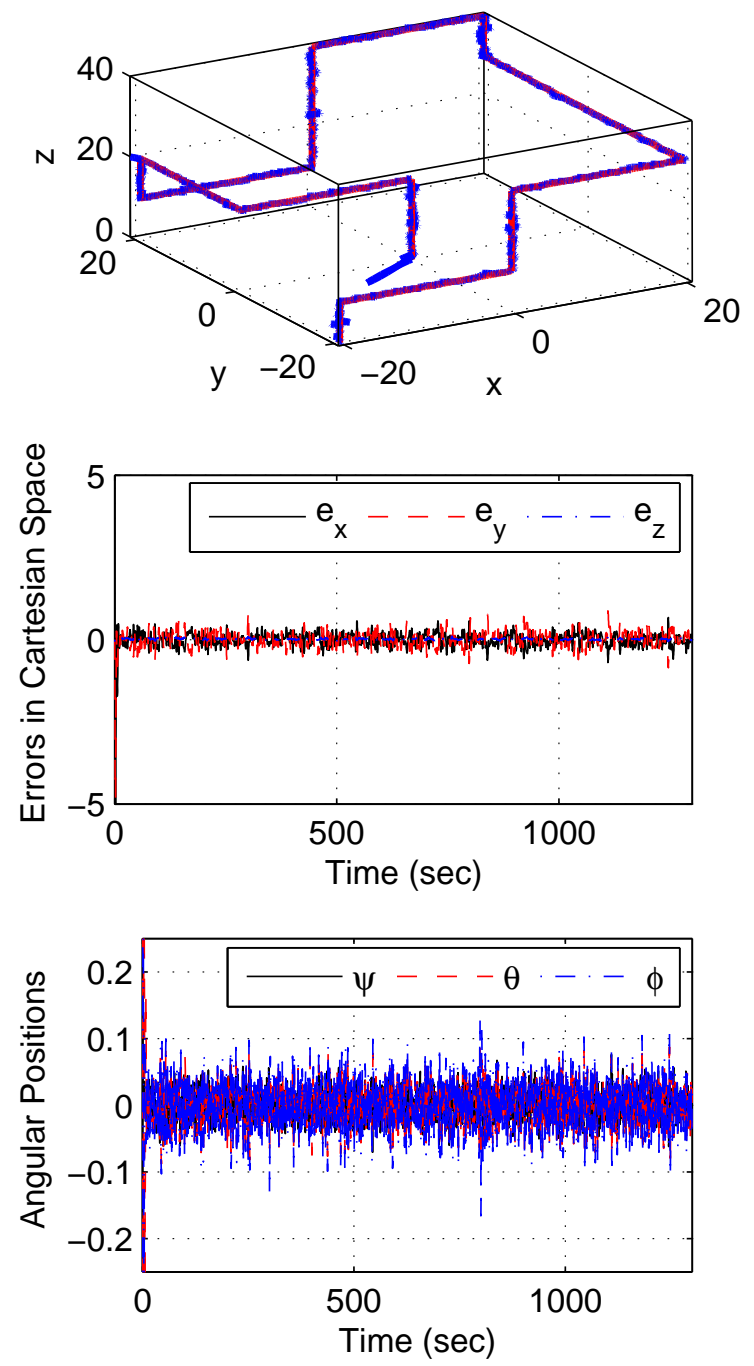

Fig. 3. Behavior in the cartesian space
Desired (Solid) Position and Vehicle Position
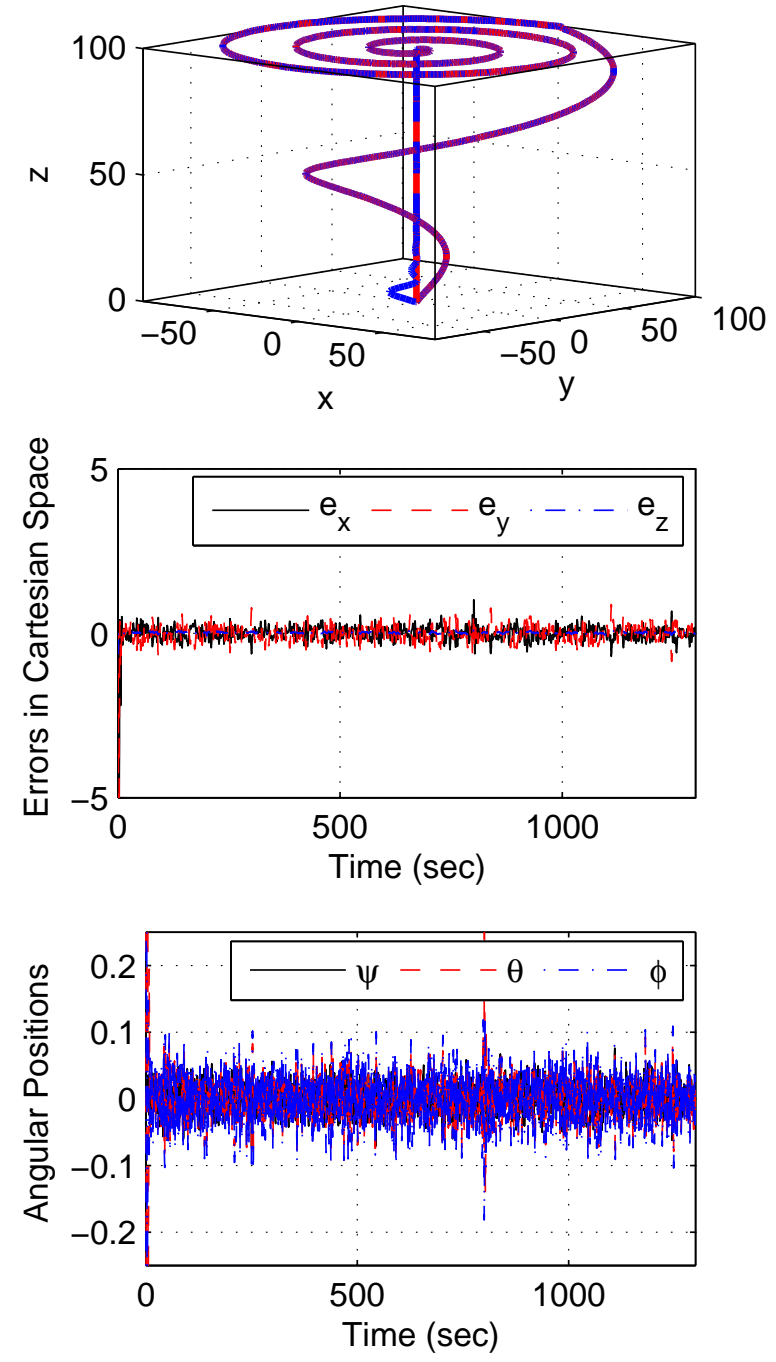

Fig. 4. Behavior in the cartesian space for the continuous trajectory of take off and landing 